\title{
Solution to Two-Dimensional Steady Inverse Heat Transfer Problems with Interior Heat Source Based on the Conjugate Gradient Method
}

\author{
Shoubin Wang, ${ }^{1}$ Li Zhang, ${ }^{1}$ Xiaogang Sun, ${ }^{2}$ and Huangchao Jia ${ }^{1}$ \\ ${ }^{1}$ School of Control and Mechanical Engineering, Tianjin Chengjian University, Tianjin 300384, China \\ ${ }^{2}$ School of Electrical Engineering and Automation, Harbin Institute of Technology, Harbin 150001, China \\ Correspondence should be addressed to Shoubin Wang; wsbin800@126.com
}

Received 5 February 2017; Revised 14 May 2017; Accepted 28 May 2017; Published 11 July 2017

Academic Editor: Francisco Alhama

Copyright (C) 2017 Shoubin Wang et al. This is an open access article distributed under the Creative Commons Attribution License, which permits unrestricted use, distribution, and reproduction in any medium, provided the original work is properly cited.

\begin{abstract}
The compound variable inverse problem which comprises boundary temperature distribution and surface convective heat conduction coefficient of two-dimensional steady heat transfer system with inner heat source is studied in this paper applying the conjugate gradient method. The introduction of complex variable to solve the gradient matrix of the objective function obtains more precise inversion results. This paper applies boundary element method to solve the temperature calculation of discrete points in forward problems. The factors of measuring error and the number of measuring points zero error which impact the measurement result are discussed and compared with L-MM method in inverse problems. Instance calculation and analysis prove that the method applied in this paper still has good effectiveness and accuracy even if measurement error exists and the boundary measurement points' number is reduced. The comparison indicates that the influence of error on the inversion solution can be minimized effectively using this method.
\end{abstract}

\section{Introduction}

Inverse heat transfer problem (Inverse Heat Transfer Problems, IHTP) means inversion unknown characteristic parameters of heat conduction objects using the internal or surface local heat measurement $[1,2]$, such as thermal physical parameters, thermal boundary conditions, geometric boundary shape, and heat conduction coefficient. Inverse heat conduction problem has a broad application prospect in nondestructive testing, geometry optimization, aerospace engineering, power engineering, mechanical engineering, construction engineering, biological engineering, metallurgical engineering, materials processing, biomedical and food engineering, and other fields [1-12]. Focusing on this problem, domestic and foreign scholars have done a lot of research. Zhu et al. studied the disadvantages of optimization algorithms and inherent space distribution characteristics of the measurement in inverse heat conduction problems. They proposed a decentralized fuzzy reasoning mechanism and established a decentralized fuzzy inference system of two-dimensional steady inverse heat conduction problems $[3,4]$. Cui et al. gave an improved conjugate gradient method. They introduced the complex variable derivation into the traditional conjugate gradient method, calculated the sensitivity coefficients accurately, and identified the boundary conditions [5]. Yu combined the boundary element method with complex variables derivation to inverse the inhomogeneous material coefficients of thermal conductivity [6]. Wang et al. applied particle swarm optimization algorithm and the least square method for solving the inverse problems. That improved the precision and shorted the solving time at the same time [13]. Yaparova studied the heat conduction boundary value inverse problem by solving the stability boundary based on Laplace and Fourier transformation [14]. Tian combined SPSO algorithm with the conjugate gradient method and the fast convergence of the traditional regularized gradient algorithm and global convergence of the intelligent optimization algorithm were highlighted [15]. Using the boundary element method, Zhou et al. analyzed two-dimensional transient conduction problems, introduced 
the conjugate gradient method to find the heat conduction coefficient, and verified the validity and stability of the method [16]. A stable differential method was proposed to solve the inverse heat conduction problems by Baranov et al. on the basis of the differential transform [17]. In this paper, the two-dimensional steady forward problems with internal heat source are solved using the boundary element method. The existing surface convective thermal dissipation is analyzed, and the conjugate gradient method is applied to solve the inverse problems. And the complex variable derivation method is introduced to increase the sensitivity coefficient and the accuracy in the inversion.

\section{Thermal Steady State Forward Problems}

2.1. The Boundary Integral Equation. The mathematical model of two-dimensional steady heat conduction problems with internal heat source is

$$
\begin{aligned}
\frac{\partial^{2} T}{\partial x^{2}}+\frac{\partial^{2} T}{\partial y^{2}} & =f \quad \in \Omega, \\
T & =\bar{T} \quad \in \Gamma_{1}, \\
\frac{\partial T}{\partial n} & =\bar{q} \quad \in \Gamma_{2}, \\
\frac{\partial T}{\partial n}+\frac{h}{\lambda} T & =\overline{q^{\prime \prime}} \quad \in \Gamma_{3},
\end{aligned}
$$

where $\Gamma_{1}, \Gamma_{2}$, and $\Gamma_{3}$ are the boundary of the domain $\Omega, \Gamma=$ $\Gamma_{1}+\Gamma_{2}+\Gamma_{3}, f=-s / \lambda, s$ is the internal heat source, $h$ is the heat conduction coefficient between solid and fluid, $\lambda=\mathrm{HTC}, T=$ temp, and $n$ is the normal vector outside the boundary. $\overline{q^{\prime \prime}}=$ $(h / \lambda) T_{f}, T_{f}$ is environment temperature, and $\bar{q}$ is heat flux.

Weight function $T^{*}$ is introduced into weighted residual of control equation.

$$
\begin{aligned}
\int_{\Omega}\left(\nabla^{2} T-f\right) T^{*} d_{\Omega}= & \int_{\Gamma_{3}}\left(\frac{\partial T}{\partial n}+\frac{h}{\lambda} T-\overline{q^{\prime \prime}}\right) T^{*} d_{\Gamma} \\
& +\int_{\Gamma_{2}}\left(\frac{\partial T}{\partial n}-\bar{q}\right) T^{*} d_{\Gamma} \\
& -\int_{\Gamma_{1}}(T-\bar{T}) \frac{\partial T^{*}}{\partial n} d_{\Gamma}
\end{aligned}
$$

Green's function of Laplace equation is $\iint_{D}\left(v \nabla^{2} u-\right.$ $\left.u \nabla^{2} v\right) d_{\Omega}=\int_{c}(v(\partial u / \partial n)-u(\partial v / \partial n)) d_{s}$, where $C$ is the boundary curve of plane closed area $D$ and $d_{s}$ is arc differential [18].

To decompose the left side of the equation, it becomes

$$
\int_{\Omega}\left(\nabla^{2} T-f\right) T^{*} d_{\Omega}=\int_{\Omega}\left(\nabla^{2} T\right) T^{*} d_{\Omega}-\int_{\Omega} f T^{*} d_{\Omega} .
$$

According to Laplace Green's function, we can have

$$
\begin{aligned}
\int_{\Omega}\left(\nabla^{2} T\right) T^{*} d_{\Omega}= & \int_{\Omega}\left(T^{*} \nabla^{2} T-T \nabla^{2} T^{*}\right) d_{\Omega} \\
& +\int_{\Omega} T \nabla^{2} T^{*} d_{\Omega} .
\end{aligned}
$$

Further reduction gives

$$
\begin{aligned}
\int_{\Omega}\left(\nabla^{2} T\right) T^{*} d_{\Omega}= & \int_{\Gamma}\left(T^{*} \frac{\partial T}{\partial n}-T \frac{\partial T^{*}}{\partial n}\right) d_{\Gamma} \\
& +\int_{\Omega} T \nabla^{2} T^{*} d_{\Omega} .
\end{aligned}
$$

Substituting (5) for the same term in (3), (2) becomes

$$
\begin{gathered}
\int_{\Gamma}\left(T^{*} \frac{\partial T}{\partial n}-T \frac{\partial T^{*}}{\partial n}\right) d_{\Gamma}+\int_{\Omega} T \nabla^{2} T^{*} d_{\Omega}-\int_{\Omega} f T^{*} d_{\Omega} \\
=\int_{\Gamma_{3}}\left(\frac{\partial T}{\partial n}+\frac{h}{\lambda} T-\overline{q^{\prime \prime}}\right) T^{*} d_{\Gamma} \\
+\int_{\Gamma_{2}}\left(\frac{\partial T}{\partial n}-\bar{q}\right) T^{*} d_{\Gamma}-\int_{\Gamma_{1}}(T-\bar{T}) \frac{\partial T^{*}}{\partial n} d_{\Gamma}
\end{gathered}
$$

Further reduction gives

$$
\begin{aligned}
\int_{\Omega} T \nabla^{2} T^{*} d_{\Omega}= & \int_{\Gamma_{3}}\left(\frac{\partial T}{\partial n}+\frac{h}{\lambda} T-\overline{q^{\prime \prime}}\right) T^{*} d_{\Gamma} \\
& +\int_{\Gamma_{2}}\left(\frac{\partial T}{\partial n}-\bar{q}\right) T^{*} d_{\Gamma} \\
& -\int_{\Gamma_{1}}(T-\bar{T}) \frac{\partial T^{*}}{\partial n} d_{\Gamma} \\
& -\int_{\Gamma}\left(T^{*} \frac{\partial T}{\partial n}-T \frac{\partial T^{*}}{\partial n}\right) d_{\Gamma} \\
& +\int_{\Omega} f T^{*} d_{\Omega} .
\end{aligned}
$$

$T^{*}$ is the basic solution of Laplace equation and it can make $\nabla T^{*}+\delta\left(r-r_{i}\right)=0$, where the point " $i$ " refers to the unit point concentration. Because $\partial T^{*} / \partial n=q^{*}$ and $\partial T / \partial n=q$, (7) can be further reduced.

The left side of (7) becomes

$$
\begin{aligned}
\int_{\Omega} T \nabla^{2} T^{*} d_{\Omega} \\
\quad=\int_{\Omega} T\left(\nabla^{2} T^{*}+\delta\left(r-r_{i}\right)\right)-T \delta\left(r-r_{i}\right) d_{\Omega} .
\end{aligned}
$$

Calculating furthermore, it is shown as follows:

$$
\begin{aligned}
& \int_{\Omega} T\left(\nabla^{2} T^{*}+\delta\left(r-r_{i}\right)\right)-T \delta\left(r-r_{i}\right) d_{\Omega} \\
& \quad=0-\int_{\Omega} T \delta\left(r-r_{i}\right) d_{\Omega} .
\end{aligned}
$$

For the selectivity of $\delta$ function, $\int_{-\infty}^{+\infty} f(x) \delta\left(x-x_{0}\right) d_{r}=$ $f\left(x_{0}\right)$. Then the reduced result on the left side of $(7)$ is

$$
-\int_{\Omega} T \delta\left(r-r_{i}\right) d_{\Omega}=-T_{i}
$$


Substituting (10) into (7),

$$
\begin{aligned}
-T_{i}= & \int_{\Gamma_{3}}\left(q+\frac{h}{\lambda} T-\overline{q^{\prime \prime}}\right) T^{*} d_{\Gamma}+\int_{\Gamma_{2}}(q-\bar{q}) T^{*} d_{\Gamma} \\
& -\int_{\Gamma_{1}}(T-\bar{T}) q^{*} d_{\Gamma}-\int_{\Gamma}\left(T^{*} q-T q^{*}\right) d_{\Gamma} \\
& +\int_{\Omega} f T^{*} d_{\Omega} .
\end{aligned}
$$

Because $\Gamma=\Gamma_{1}+\Gamma_{2}+\Gamma_{3}, \int_{\Gamma}\left(T^{*} q-T q^{*}\right) d_{\Gamma}$ is decomposed:

$$
\begin{aligned}
\int_{\Gamma}\left(T^{*} q-T q^{*}\right) d_{\Gamma}= & \int_{\Gamma_{1}}\left(T^{*} q-T q^{*}\right) d_{\Gamma} \\
& +\int_{\Gamma_{2}}\left(T^{*} q-T q^{*}\right) d_{\Gamma} \\
& +\int_{\Gamma_{3}}\left(T^{*} q-T q^{*}\right) d_{\Gamma}
\end{aligned}
$$

Substituting (12) for the same term in (11),

$$
\begin{aligned}
-T_{i}= & \int_{\Gamma_{3}}\left(\frac{h}{\lambda} T T^{*}-\overline{q^{\prime \prime}} T^{*}+T q^{*}\right) d_{\Gamma} \\
& +\int_{\Gamma_{2}}\left(T q^{*}-\bar{q} T^{*}\right) d_{\Gamma}+\int_{\Gamma_{1}}\left(\bar{T} q^{*}-T^{*} q\right) d_{\Gamma} \\
& +\int_{\Omega} f T^{*} d_{\Omega} .
\end{aligned}
$$
makes

$$
\begin{aligned}
-T_{i}= & \int_{\Gamma_{3}} \frac{h}{\lambda} T T^{*} d_{\Gamma} \\
& +\left(\int_{\Gamma_{3}} T q^{*} d_{\Gamma}+\int_{\Gamma_{2}} T q^{*} d_{\Gamma}+\int_{\Gamma_{1}} \bar{T} q^{*} d_{\Gamma}\right) \\
& -\left(\int_{\Gamma_{3}} \overline{q^{\prime \prime}} T^{*} d_{\Gamma}+\int_{\Gamma_{2}} \bar{q} T^{*} d_{\Gamma}+\int_{\Gamma_{1}} q T^{*} d_{\Gamma}\right) \\
& +\int_{\Omega} f T^{*} d_{\Omega} .
\end{aligned}
$$

The second and the third terms are combined, which

$$
\begin{aligned}
T_{i} & +\int_{\Gamma} q^{*} T d_{\Gamma}+\int_{\Gamma_{3}} \frac{h}{\lambda} T T^{*} d_{\Gamma} \\
& =\int_{\Gamma} T^{*} q d_{\Gamma}-\int_{\Omega} f T^{*} d_{\Omega} .
\end{aligned}
$$

The two-dimensional steady $T^{*}=(1 / 2 \pi) \ln (1 / r), \quad r=$ $\sqrt{\left(x-x_{i}\right)^{2}-\left(y-y_{i}\right)^{2}}$.

Moving the source point $i$ to the boundary, the integral equation at any point on the boundary can be obtained. Thus, the basic solution $T^{*}$ and the singularity of the integral $\partial_{T^{*}} / \partial_{n}$ need to be considered. Assuming that a circular arc centering on the point $i$ near the border and $\varepsilon$ is the small radius of the circle, (15) can be written as

$$
\begin{array}{r}
T_{i}+\int_{\Gamma-\varepsilon} q^{*} T d_{\Gamma}+\int_{\Gamma \varepsilon} q^{*} T d_{\Gamma}+\int_{\Gamma_{3}} \frac{h}{\lambda} T T^{*} d_{\Gamma} \\
=\int_{\Gamma-\varepsilon} T^{*} q d_{\Gamma}+\int_{\Gamma \varepsilon} T^{*} q d_{\Gamma}-\int_{\Omega} f T^{*} d_{\Omega},
\end{array}
$$

where $\Gamma \varepsilon$ is the new circular border and $\Gamma-\varepsilon$ is the boundary outside the new circular boundary in $\Gamma$. Considering the smooth boundary, the integral mean value theorem is introduced.

$$
\begin{aligned}
\int_{\Gamma \varepsilon} T q^{*} d_{\Gamma} & =\int_{\Gamma \varepsilon} T \frac{\partial}{\partial n}\left(\frac{1}{4 \pi \varepsilon^{2}}\right) d_{\Gamma}=\int_{\Gamma \varepsilon} T \frac{-1}{4 \pi \varepsilon^{2}} d_{\Gamma} \\
& =T(\xi) \frac{-1}{4 \pi \varepsilon^{2}} 2 \pi \varepsilon^{2},
\end{aligned}
$$

where $\xi$ is the point on $\Gamma \varepsilon$. Let $\varepsilon \rightarrow 0 T(\xi) \rightarrow T_{i}$; then

$$
\begin{aligned}
\lim _{\varepsilon \rightarrow 0}\left(\int_{\Gamma \varepsilon} T q^{*} d_{\Gamma}\right) & =-\frac{1}{2} T_{i}, \\
\lim _{\varepsilon-0}\left(\int_{\Gamma-\varepsilon} q^{*} T d_{\Gamma}\right) & =\int_{\Gamma} q^{*} T d_{\Gamma}, \\
\lim _{\varepsilon-0}\left(\int_{\Gamma-\varepsilon} q T^{*} d_{\Gamma}\right) & =\int_{\Gamma} q^{*} T d_{\Gamma} .
\end{aligned}
$$

Synthesizing the above limit results, we have

$$
\begin{gathered}
\frac{1}{2} T_{i}+\int_{\Gamma} q^{*} T d_{\Gamma}+\int_{\Gamma_{3}} \frac{h}{\lambda} T T^{*} d_{\Gamma} \\
=\int_{\Gamma} T^{*} q d_{\Gamma}-\int_{\Omega} f T^{*} d_{\Omega} .
\end{gathered}
$$

Considering (15) and (19) simultaneously, boundary integral equation of any point in the domain $\Omega$ or on the boundary can be found out:

$$
\begin{gathered}
C_{i} T_{i}+\int_{\Gamma} q^{*} T d_{\Gamma}+\int_{\Gamma_{3}} \frac{h}{\lambda} T T^{*} d_{\Gamma} \\
=\int_{\Gamma} T^{*} q d_{\Gamma}-\int_{\Omega} f T^{*} d_{\Omega}, \\
C_{i}= \begin{cases}1 & \in \Omega, \\
\frac{1}{2} & \in \Gamma .\end{cases}
\end{gathered}
$$

2.2. Calculation of the Unknown Value Based on Boundary Element Method. Discreteness of boundary integral equations: because the quantities $T$ and $q$ on the boundary are known, the only thing to do is divide the boundary discretely. Boundary $\Gamma$ is divided into $N$ boundary units $\Gamma_{j}(j=$ $1, \ldots, N)$, where there are $N_{1}$ units on the boundary $\Gamma_{1}, N_{2}$ units on the boundary $\Gamma_{2}$, and $N_{3}$ units on the boundary $\Gamma_{3}$. $N=N_{1}+N_{2}+N_{3}$. 
The unknown points in the boundary units are called "nodes." For the requirements of different difference, the expressions of $T$ and $q$ to be found out can be written as

$$
\begin{gathered}
T=\sum_{i=1}^{N} \varphi_{i}(\xi) T_{i}, \\
q=\sum_{i=1}^{N} \varphi_{i}(\xi) q_{i},
\end{gathered}
$$

where $N$ is the interpolation node number in a boundary unit and $\varphi$ is the interpolation function. When $j$ is in $\Gamma_{1}$ or $\Gamma_{2},(19)$ can be written as

$$
\begin{aligned}
\frac{1}{2} T_{i} & +\sum_{j=1}^{N_{1}+N_{2}} \int_{\Gamma j} q^{*} T d_{\Gamma} \\
= & \sum_{j=1}^{N_{1}+N_{2}} \int_{\Gamma j} T^{*} q d_{\Gamma}-\int_{\Omega} f T^{*} d_{\Omega} .
\end{aligned}
$$

This paper uses linear interpolation. Because $\varphi_{1}(\xi)=(1-\xi) /$ $2, \varphi_{2}(\xi)=(1+\xi) / 2$ is the interpolation function of linear unit, the straight line is used to approximate the boundary curve, and the two linearized terminal values are used to approximate the values of $T$ and $q$. So the node values can be converted into the following:

$$
\begin{aligned}
T(\xi) & =\varphi_{1}(\xi) T_{j}+\varphi_{2}(\xi) T_{j+1} \\
& =\left[\varphi_{1}(\xi), \varphi_{2}(\xi)\right]\left(\begin{array}{c}
T_{j} \\
T_{j+1}
\end{array}\right), \\
q(\xi) & =\varphi_{1}(\xi) q_{j}+\varphi_{2}(\xi) q_{j+1} \\
& =\left[\varphi_{1}(\xi), \varphi_{2}(\xi)\right]\left(\begin{array}{c}
q_{j} \\
q_{j+1}
\end{array}\right) .
\end{aligned}
$$

Substituting (23a) and (23b) into (22),

$$
\begin{aligned}
\frac{1}{2} T_{i} & +\sum_{j=1}^{N_{1}+N_{2}}\left(h_{i j}{ }^{(1)} T_{j}+h_{i j}{ }^{(2)} T_{j+1}\right) \\
= & \sum_{j=1}^{N_{1}+N_{2}}\left(g_{i j}{ }^{(1)} q_{j}+g_{i j}{ }^{(2)} q_{j+1}\right)-\int_{\Omega} f T^{*} d_{\Omega},
\end{aligned}
$$

where

$$
\begin{aligned}
& h_{i j}{ }^{(e)}=\int_{\Gamma j} \varphi_{e} q^{*} d_{\Gamma}, \\
& g_{i j}{ }^{(e)}=\int_{\Gamma j} \varphi_{e} T^{*} d_{\Gamma}, \\
& e=(1,2) .
\end{aligned}
$$

Let

$$
\begin{aligned}
& H_{i j}=h_{i j-1}{ }^{(2)}+h_{i j}{ }^{(1)}, \\
& G_{i j}=g_{i j-1}{ }^{(2)}+g_{i j}{ }^{(1)},
\end{aligned}
$$

and we have

$$
\frac{1}{2} T_{i}+\sum_{j=1}^{N_{1}+N_{2}} H_{i j} T_{j}=\sum_{j=1}^{N_{1}+N_{2}} G_{i j} q_{j}-\int_{\Omega} f T^{*} d_{\Omega} .
$$

Similarly, when the point locates in $\Gamma_{3}$, (24) becomes

$$
\begin{aligned}
& \frac{1}{2} T_{i}+\sum_{j=1}^{N_{3}}\left[\left(h_{i j}{ }^{(1)}+\frac{h}{\lambda} g_{i j}{ }^{(1)}\right) T_{j}\right. \\
& \left.+\left(h_{i j}{ }^{(2)}+\frac{h}{\lambda} g_{i j}{ }^{(2)}\right) T_{j+1}\right]=\sum_{j=1}^{N_{3}}\left(g_{i j}{ }^{(1)} q_{j}\right. \\
& \left.+g_{i j}{ }^{(2)} q_{j+1}\right)-\int_{\Omega} f T^{*} d_{\Omega} .
\end{aligned}
$$

Let

$$
\begin{aligned}
& H_{i j}=\left(h_{i j-1}{ }^{(2)}+\frac{h}{\lambda} g_{i j-1}{ }^{(2)}\right)+\left(h_{i j}{ }^{(1)}+\frac{h}{\lambda} g_{i j}{ }^{(1)}\right), \\
& G_{i j}=g_{i j-1}{ }^{(2)}+g_{i j}{ }^{(1)},
\end{aligned}
$$

and the boundary integral equation is

$$
\frac{1}{2} T_{i}+\sum_{j=1}^{N_{3}} H_{i j} T_{j}=\sum_{j=1}^{N_{3}} G_{i j} q_{j}-\int_{\Omega} f T^{*} d_{\Omega} .
$$

Rearranging (30), we have

$$
\sum_{j=1}^{N} \widehat{H}_{i j} T_{j}=\sum_{j=1}^{N} G_{i j} q_{j}-\int_{\Omega} f T^{*} d_{\Omega}
$$

where

$$
\widehat{H}_{i j}= \begin{cases}H_{i j}, & (i \neq j), \\ H_{i j}+\frac{1}{2}, & (i=j)\end{cases}
$$

considering $B_{i}=\int_{\Omega} f T^{*} d_{\Omega}$, (31) can be expressed as a matrix form $H T=G Q-B_{i}$. The boundary conditions $T$ and $Q$ are written as $T=\left[\begin{array}{l}\bar{T}_{1} \\ T_{2}\end{array}\right], Q=\left[\begin{array}{l}Q_{1} \\ \bar{Q}_{2}\end{array}\right]$, where $\bar{T}_{1}, \bar{Q}_{2}$ are known temperature and heat flux. Equation (31) becomes

$$
\left[\begin{array}{ll}
H_{1} & H_{2}
\end{array}\right]\left[\begin{array}{l}
\bar{T}_{1} \\
T_{2}
\end{array}\right]=\left[\begin{array}{ll}
G_{1} & G_{2}
\end{array}\right]\left[\begin{array}{l}
Q_{1} \\
\bar{Q}_{2}
\end{array}\right]-B_{i} .
$$

Put the known quantities on the left.

$$
\left[\begin{array}{ll}
H_{2} & -G_{1}
\end{array}\right]\left[\begin{array}{c}
T_{2} \\
Q_{1}
\end{array}\right]=\left[\begin{array}{ll}
G_{2} & -H_{1}
\end{array}\right]\left[\begin{array}{l}
\bar{Q}_{2} \\
\bar{T}_{1}
\end{array}\right]-B_{i} .
$$

Appling the radial integration method, domain integration caused by heat source is converted to boundary integration [19-21].

$$
B_{i}=\int_{\Omega} f T^{*} d_{\Omega}=\int_{\Gamma} \frac{F}{R} \frac{\partial r}{\partial n} d_{\Gamma}
$$


where $R$ is the distance between the source and the field point and $F$ is radial integration which is expressed as

$$
F=\int_{0}^{R} f T^{*} r d_{r}
$$

Because $f$ is a known function and the function form is simple, $T^{*}$ is the basic function, the radial integration can be found out through (36), and the boundary integration can be obtained from (35).

Thus, (34) can be written as $A X=K$ to solve the above equations and to find $T$ and $Q$.

\section{The Steady Inverse Problem}

3.1. The Objective Function of Inverse Problem. For aforementioned heat conduction system, the heat transfer coefficient between solid and fluid $h$ and the thermal conductivity $\lambda$ are known. The boundary temperature to be found out is unknown which can be determined based on the known measured boundary temperature and the known condition of forward problem.

The following objective function is defined as

$$
J(q)=\sum_{i=1}^{N}\left[T_{i}(q)-T_{i}^{\mathrm{mea}}\right]^{2}
$$

In the objective function, $q$ is a parameter vector whose temperature needs to be inversed and $N$ is the number of temperature measuring points on the boundary.

$$
q=\left\{r_{1}, r_{2}, \ldots, r_{m}\right\}=\left\{T_{11}, T_{21}, \ldots, T_{m 1}\right\} .
$$

$T_{i}(q)$ is the calculation of measuring point $i$ in the forward problem and $T^{\text {mea }}$ is the measurement of temperature. The minimum of the objective function $J(q)$ is the parameter vector $q$ of inverse problem.

3.2. Conjugate Gradient Method of Inverse Problem. Conjugate gradient method is a method which combines the conjugacy and the steepest descent method. It derives from perturbation principle and the inverse problem is converted into three questions, such as forward problem, sensitivity problem, and adjoint problem. In order to solve the effects of these three questions, this paper introduces the complex variable derivation method into the traditional conjugate gradient method, which makes the calculation of the sensitivity coefficient accurate.

During the calculation, unconstrained optimization algorithm is achieved by iteration. Considering that the $k$ th iteration point $q_{k}$ has been available, the $(k+1)$ th iteration calculates according to the following formula.

The iterative equation solving the inverse problem by conjugate gradient method includes

$$
\begin{aligned}
q_{k+1} & =q_{k}+\alpha_{k} d_{k}, \\
q^{k+1} & =q^{k}-\alpha^{k} d^{k}, \\
d_{k} & = \begin{cases}-g_{k}, & k=0, \\
-g_{k}+\beta_{k} d_{k-1}, & k \geq 1,\end{cases}
\end{aligned}
$$

where $\alpha_{k}$ is step length, obtained by searching some onedimensional line; $d_{k}$ is the searching direction in which $g_{k}=$ $\nabla J\left(q_{k}\right)$ and $\beta_{k}$ is a scalar. Different $\beta_{k}$ corresponds to different nonlinear conjugate gradient methods.

The searching step length $\alpha^{k}$, the conjugate coefficient $\beta^{k}$, and gradient $\nabla J\left(q^{k}\right)$ are needed to be found out.

Conjugate coefficient $\beta^{k}$ equals the ratio of the square of normal form between the current and the previous-stepgradient paradigm:

$$
\begin{aligned}
& \beta_{k}=\frac{\left[\nabla J\left(q_{k}\right)-\nabla J\left(q_{k-1}\right)\right] \nabla J^{T}\left(q_{k}\right)}{\nabla J\left(q_{k-1}\right) \nabla J^{T}\left(q_{k-1}\right)}, \\
& \nabla J\left(q_{k}\right)=\left(\frac{\partial J}{\partial r_{1}}, \frac{\partial J}{\partial r_{2}}, \ldots, \frac{\partial J}{\partial r_{m+1}}\right), \\
& \frac{\partial J\left(q_{k}\right)}{\partial r_{j}}=2 \sum_{i=1}^{N}\left(T_{i} q_{k}-T_{i}^{*}\right) \frac{\partial T_{i}\left(q_{k}\right)}{\partial r_{j}} \\
& \quad j=1,2, \ldots, m+1 .
\end{aligned}
$$

If the iteration step is $k+1$ and searching step length is $\alpha_{k}$, (37) $J\left[q_{k+1}\right]=\sum_{i=1}^{N}\left[T_{i}\left(q_{k}+\alpha_{k} d_{k}\right)-T^{*}\right]^{2}$ gives

$$
\alpha_{k}=\frac{\sum_{i=1}^{N}\left[T_{i}\left(q_{k}\right)-T_{i}^{*}\right] \nabla T_{i} d_{k}}{\sum_{i=1}^{N}\left[\nabla T_{i} d_{k}\right]^{2}},
$$

where $\nabla T_{i}=\left(\partial T_{i} / \partial_{r 1}, \partial T_{i} / \partial_{r 2}, \ldots, \partial T_{i} / \partial_{r m+1}\right)$.

3.3. Complex Variable Derivation Method [22-24]. For any real function $f(x)$, a very small imaginary part $h$ is added to the real variable $x$. It is expressed in complex function $f(x+i h)$ and its Taylor series is

$$
f(x+i h)=f(x)+i h f^{\prime}(x)-\frac{h^{2}}{2} f^{\prime \prime}(x)+o\left(h^{3}\right) .
$$

As $h$ is very small,

$$
\begin{aligned}
\frac{d f}{d x} & =\frac{\operatorname{Im}(f(x+i h))}{h} \\
\frac{d^{2} f}{d x^{2}} & =\frac{2[f(x)-\operatorname{Re}(f(x+i h))]}{h^{2}}
\end{aligned}
$$

where $\partial T_{i}\left(q_{k}\right) / \partial r_{j}$ is sensitivity coefficient and its matrix form is

$$
\frac{\partial T}{\partial r}=\left[\begin{array}{cccc}
\frac{\partial T_{1}(r)}{\partial r_{1}} & \frac{\partial T_{1}(r)}{\partial r_{2}} & \cdots & \frac{\partial T_{1}(r)}{\partial r_{m}} \\
\frac{\partial T_{2}(r)}{\partial r_{1}} & \frac{\partial T_{2}(r)}{\partial r_{2}} & \cdots & \frac{\partial T_{2}(r)}{\partial r_{m}} \\
\vdots & \vdots & \vdots & \vdots \\
\frac{\partial T_{N}(r)}{\partial r_{1}} & \frac{\partial T_{N}(r)}{\partial r_{2}} & \cdots & \frac{\partial T_{N}(r)}{\partial r_{m}}
\end{array}\right]
$$

The complex variable derivation method is used to calculate the matrix of sensitivity coefficient. 


$$
\frac{\partial T}{\partial r}=\left[\begin{array}{cccc}
\frac{\operatorname{Im}\left(T_{1}\left(r_{1}+h\right)\right)}{h} & \frac{\operatorname{Im}\left(T_{1}\left(r_{2}+h\right)\right)}{h} & \cdots & \frac{\operatorname{Im}\left(T_{1}\left(r_{m}+h\right)\right)}{h} \\
\frac{\operatorname{Im}\left(T_{2}\left(r_{1}+h\right)\right)}{h} & \frac{\operatorname{Im}\left(T_{2}\left(r_{2}+h\right)\right)}{h} & \cdots & \frac{\operatorname{Im}\left(T_{2}\left(r_{m}+h\right)\right)}{h} \\
\vdots & \vdots & \vdots & \vdots \\
\frac{\operatorname{Im}\left(T_{N}\left(r_{1}+h\right)\right)}{h} & \frac{\operatorname{Im}\left(T_{N}\left(r_{2}+h\right)\right)}{h} & \cdots & \frac{\operatorname{Im}\left(T_{N}\left(r_{m}+h\right)\right)}{h}
\end{array}\right] .
$$

The complex variable derivation method is introduced into the traditional conjugate gradient method, which makes the calculation of the sensitivity coefficient accurate and avoids the sensitivity problem and the adjoint problem.

3.4. Solving Process of the Inverse Problem. Initialization: $k=$ $0, q_{0}, r_{0}=0$, and let $\varepsilon$ be a small positive number.

Solve the forward problem given by (1), calculate temperature $T_{i}$, and judge if the following condition is true:

$$
J(q)=\sum_{i=1}^{N}\left[T_{i}(q)-T_{i}^{\text {mea }}\right]^{2}<\varepsilon .
$$

The equation means the condition required by the instruction DO WHILE has not been satisfied.

Calculating the gradient $\nabla J$ using (41)

Calculating the searching-down direction $d_{k}$ using (27)

Calculating the searching step length $\alpha_{k}$ using (43)

Calculating the new estimation using (34)

Solving the forward problem given by (1) and obtain$\operatorname{ing} T_{i}$ :

$$
k=k+1
$$

End do

\section{The Instance Calculation and Analysis}

To testify the availability of the aforementioned method, the simulation experiment having two sets of data was designed. The experiment discussed the effect of the testing points' number and the measuring error on the inversion results and compared CGM and L-MM. The schematic diagram is shown as Figure 1.

In the experiment, the concrete slab's thickness is $\delta=$ $0.3 \mathrm{~m}$, and the thickness of casting concrete slab between two wooden templates is $\delta_{1}=\delta_{2}=0.02 \mathrm{~m}$, and coefficient of thermal conductivity is $\lambda_{1}=\lambda_{2}=0.16 \mathrm{w} /(\mathrm{mK})$, the air temperature is $T_{f}=293 \mathrm{~K}$, and $D 1$ and $D 3$ are the convective heat conduction boundary conditions. The heat transfer coefficients are $h_{i}=8 \mathrm{~W} /\left(\mathrm{m}^{2} \mathrm{~K}\right)$ and $h_{e}=20 \mathrm{~W} /\left(\mathrm{m}^{2} \mathrm{~K}\right)$. The thermal conductivity coefficient is $\lambda=1.5 \mathrm{~W} /(\mathrm{mK})$ and the power density of inner heat source is $s=1000 \mathrm{~W} / \mathrm{m}^{3}$ (similar to even-distributed and constant in short time). The real temperature distribution of the boundary $\mathrm{D} 4$ to be solved is

$$
y=311+48 x-\frac{1000 x^{2}}{3}
$$

According to the real temperatures, the calculated temperatures $T_{i}$ of testing points on the known boundary $D 2$ are counted through the forward question.

$$
\begin{aligned}
T_{i}^{\text {mea }} & =T_{i}+\omega \sigma, \\
\varepsilon & =\sum_{i=1}^{N} \sigma^{2},
\end{aligned}
$$

where $\omega$ is standard-normal-distributed random number and $\sigma$ is the standard deviation of measuring temperatures.

4.1. The Influence of Measuring Points' Number on Inversion. When the measurement error is zero, $T_{f}=293 \mathrm{~K}, h_{i}=$ $8 \mathrm{~W} /\left(\mathrm{m}^{2} \mathrm{~K}\right)$, and $h_{e}=20 \mathrm{~W} /\left(\mathrm{m}^{2} \mathrm{~K}\right)$. The thermal conductivity coefficient is $\lambda=1.5 \mathrm{~W} /(\mathrm{mK})$ and the power density of inner heat source is $s=1000 \mathrm{~W} / \mathrm{m}^{3}$. The influence of measuring points' number on inversion is shown as Figure 2. When the measuring point $N=5,9$, or 12 , the inversion results reveal that the more points are measured, the more accuracy of inversion can be achieved.

4.2. The Influence of the Measuring Error on Inversion. $N=$ $12, T_{f}=293 \mathrm{~K}, h_{i}=8 \mathrm{~W} /\left(\mathrm{m}^{2} \mathrm{~K}\right)$, and $h_{e}=20 \mathrm{~W} /\left(\mathrm{m}^{2} \mathrm{~K}\right)$. The thermal conductivity coefficient is $\lambda=1.5 \mathrm{~W} /(\mathrm{mK})$, and the power density of inner heat source is $s=1000 \mathrm{~W} / \mathrm{m}^{3}$. The influence of different measuring error on inversion is shown as Figure 3. As the measuring standard deviation $\sigma=0,0.2,0.4$, the inversion results reveal that the method can obtain better inversion results for a relatively small measurement standard deviation.

4.3. The Comparison between CGM and L-MM. This paper applies CGM and L-MM to calculate the inversion and compare with each other under three conditions: $\sigma=0$, $N=9$, and $\sigma=0, N=12$, and $N=12, \sigma=0.2$ (other conditions are the same as the aforementioned).

The inversions under the conditions $\sigma=0, N=9$, and $\sigma=0, N=12$ are shown as Figures 4 and 5 . Figures 4 and 5 


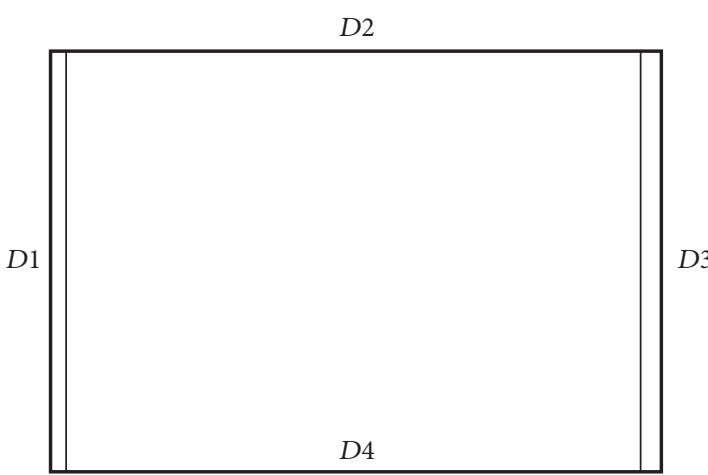

Figure 1: Model of the instance.

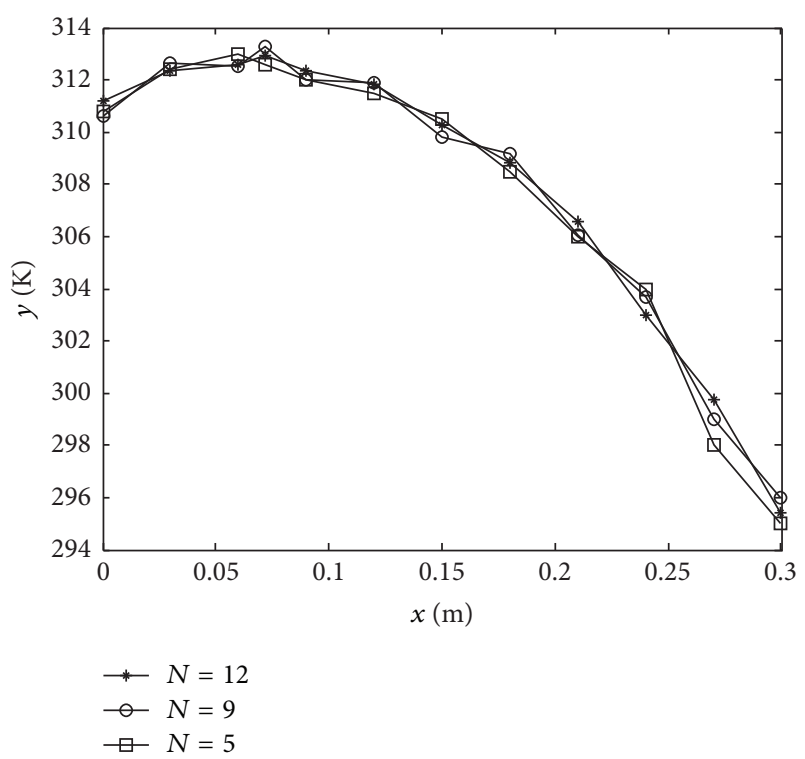

FIGURE 2: The influence of measuring points' number on inversion.

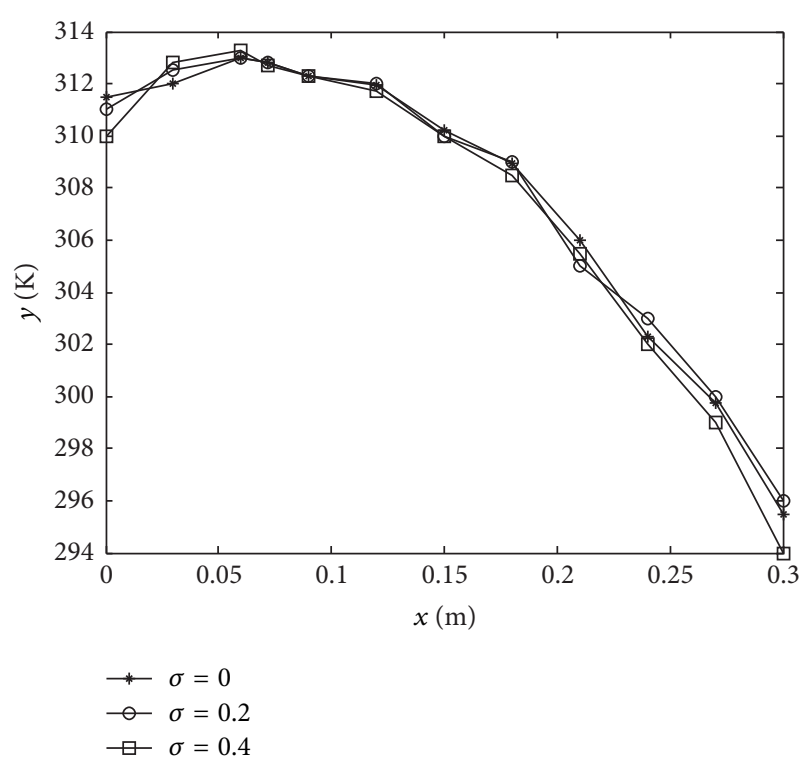

FIGURE 3: The influence of different measuring error on inversion.

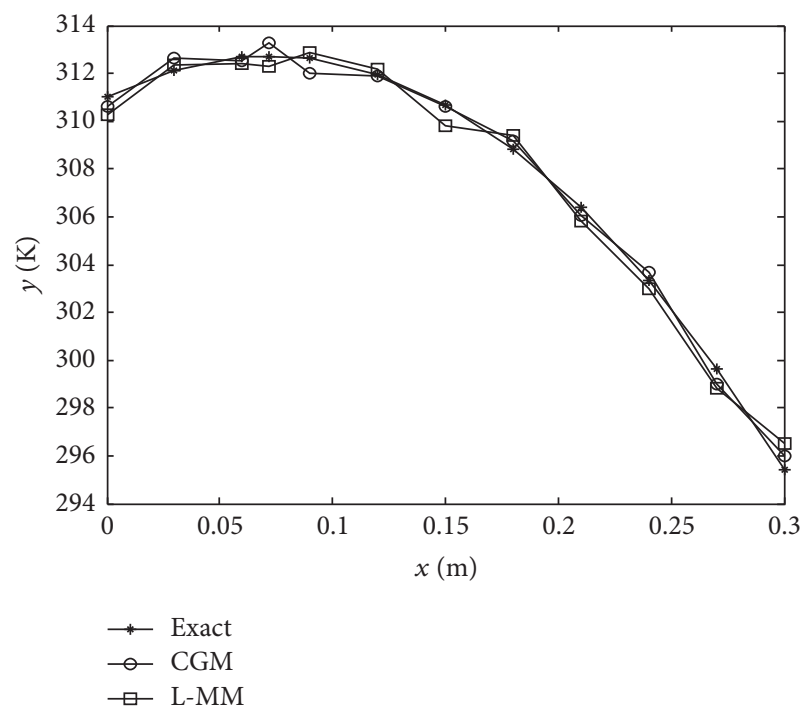

Figure 4: The inversion under the condition: $\sigma=0$ and $N=9$.

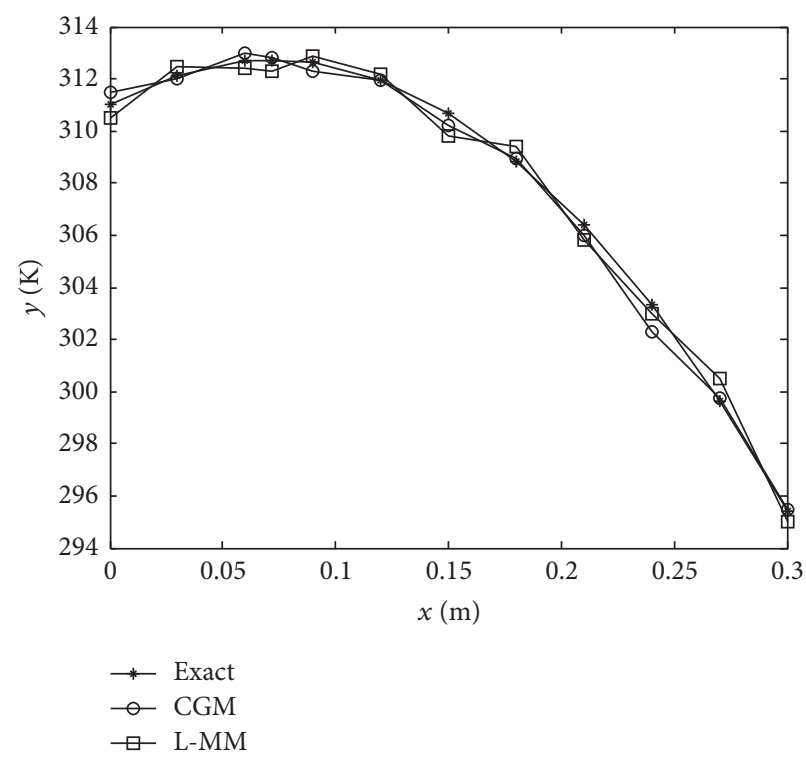

FIGURE 5: The inversion under the condition: $\sigma=0$ and $N=12$.

show that the inversions using CGM and L-MM are similar and both have high accuracy. Both of them are satisfied.

The inversions under the conditions $\sigma=0.2, N=9$ are shown as Figure 6. Comparing with Figure 5, the inversions using L-MM cause obvious fluctuation when the measuring error increases, while the inversions using CGM are satisfied. That means CGM has the better stability.

\section{Conclusion}

This paper applies CGM based on complex variable derivation to study the multivariable inverse problem which combines boundary distribution with convection coefficient of two-dimensional steady system with inner heat source. The inversions applying CGM and L-MM are compared. The 


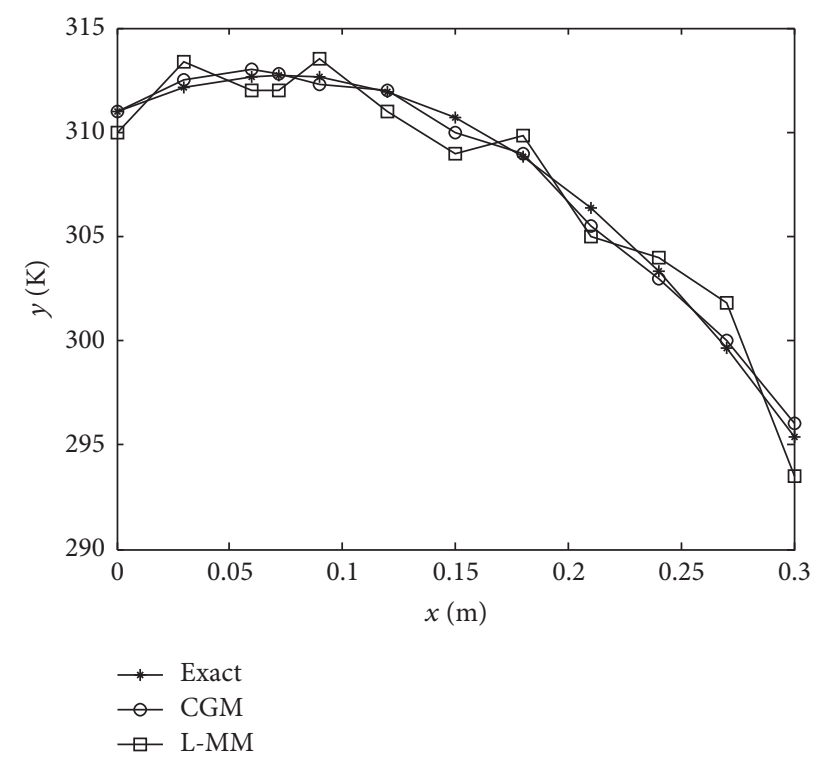

FIgURE 6: The inversion under the condition: $\sigma=0.2$ and $N=12$.

influence of measuring points' number and measuring error on inversion is tested. The simulation reveals that applying boundary element method and conjugate gradient method to solve the inverse heat conduction problem is successful and has good stability.

\section{Conflicts of Interest}

The authors declare that they have no conflicts of interest.

\section{Acknowledgments}

This work was financially supported by the National Key Foundation for Exploring Scientific Instrument of China (2013YQ470767), Tianjin Science and Technology Committee for Science and Technology Development Strategy Research Project (15ZLZLZF00350), Tianjin Science and Technology Commissioner Project (16JCTPJC53000), Science and Technology Plan Project of Tianjin Science and Technology Committee (16ZLZXZF00270), and the 13th Five-Year Plan (2016-2020) of Science Education Project in Tianjin City (HE1017).

\section{References}

[1] C. Sheng, Direct And Inverse Heat Conduction Problems Solving by The Boundary Element Method, Hunan University, 2007.

[2] K. A. Woodbury, J. V. Beck, and H. Najafi, "Filter solution of inverse heat conduction problem using measured temperature history as remote boundary condition," International Journal of Heat and Mass Transfer, vol. 72, pp. 139-147, 2014.

[3] L. Zhu, G. Wang, and H. Chen, "Estimating steady multivariables inverse heat conduction problem by using conjugate gradient method," Proceedings of the Chinese Society of Electrical Engineering, vol. 31, no. 8, pp. 58-61, 2011.

[4] L. Zhu, Fuzzy Inverse for Two-Dimensional Steady Heat Conduction System And Application, Chongqing University.
[5] M. Cui, W.-W. Duan, and X.-W. Gao, "Conjugate Gradient Method Based on Complex-variable-differentiation Method and Its Application for Identification of Boundary Conditions in Inverse Heat Conduction Problem," CIESC Journal, vol. 90, supplement 1, pp. 106-110, 2015.

[6] X. Yu, INverse Analysis of Thermal Conductivities in NonHomogeneous Heat Conductions Using Boundary Element Method, Dalian University of Technology, 2013.

[7] Z. Qian, "Optimal modified method for a fractional-diffusion inverse heat conduction problem," Inverse Problems in Science and Engineering, vol. 18, no. 4, pp. 521-533, 2010.

[8] B. T. Johansson, D. Lesnic, and T. Reeve, "A method of fundamental solutions for the radially symmetric inverse heat conduction problem," International Communications in Heat and Mass Transfer, vol. 39, no. 7, pp. 887-895, 2012.

[9] S. Tapaswini, S. Chakraverty, and D. Behera, "Numerical solution of the imprecisely defined inverse heat conduction problem," Chinese Physics B, vol. 24, no. 5, Article ID 050203, 2015.

[10] P. Duda, "Numerical and experimental verification of two methods for solving an inverse heat conduction problem," International Journal of Heat and Mass Transfer, vol. 84, pp. 1101-1112, 2015.

[11] Y. B. Wang, J. Cheng, J. Nakagawa, and M. Yamamoto, "A numerical method for solving the inverse heat conduction problem without initial value," Inverse Problems in Science and Engineering, vol. 18, no. 5, pp. 655-671, 2010.

[12] Q. Xue and w. Wei, "Parameters identification of non-linear inverse heat conduction problem," Engineering Mechanics, vol. 27, no. 8, pp. 5-9, 2010.

[13] L. Wang, L. Mei, and J. Huang, "Inverse heat conduction problem based on least squares prediction," CIESC Journal, vol. 67, supplement 1, pp. 103-110, 2016.

[14] N. Yaparova, "Numerical methods for solving a boundary-value inverse heat conduction problem," Inverse Problems in Science and Engineering, vol. 22, no. 5, pp. 832-847, 2014.

[15] N. Tian, Numerical Methods for The PDE-Based Inverse Problems and Applications, Jiangnan University, 2012.

[16] H. Zhou, X. Xu, X. Li, and H. Chen, "Identification of temperature-dependent thermal conductivity for 2-d transient heat conduction problems," Applied Mathematics and Mechanics, vol. 12, no. 35, pp. 1341-1351, 2014.

[17] V. L. Baranov, A. A. Zasyad'ko, and G. A. Frolov, "Integrodifferential method of solving the inverse coefficient heat conduction problem," Journal of Engineering Physics and Thermophysics, vol. 83, no. 1, pp. 60-71, 2010.

[18] H. Wu, Heat Conduction Problem Solving By Boundary Element Method, National Defence Industry Press, Beijing, China, 2008.

[19] L. J. M. Jesus, C. A. Cimini, and E. L. Albuquerque, "Application of the radial integration method into dynamic formulation of anisotropic shallow shells using boundary element method," Key Engineering Materials, vol. 627, pp. 465-468, 2015.

[20] X. W. Gao, "The radial integration method for evaluation of domain integrals with boundary-only discretization," Engineering Analysis with Boundary Elements, vol. 26, no. 10, pp. 905916, 2002.

[21] S. Qu, S. Li, H.-R. Chen, and Z. Qu, "Radial integration boundary element method for acoustic eigenvalue problems," Engineering Analysis with Boundary Elements, vol. 37, supplement 6-7, pp. 1043-1051, 2013. 
[22] M.-W. Liu, Y.-R. Zheng, and Y.-F. Zhang, "A new inversion method of rock-soils parameters based on complex-variabledifferentiation method," Chinese Journal of Computational Mechanics, vol. 26, no. 5, pp. 676-683, 2009.

[23] J. N. Lyness and C. B. Moler, "Numerical differentiation of analytic functions," SIAM Journal on Numerical Analysis, vol. 4, pp. 202-210, 1967.

[24] S. Kim, J. Ryu, and M. Cho, "Numerically generated tangent stiffness matrices using the complex variable derivative method for nonlinear structural analysis," Computer Methods in Applied Mechanics and Engineering, vol. 200, no. 1-4, pp. 403-413, 2011. 


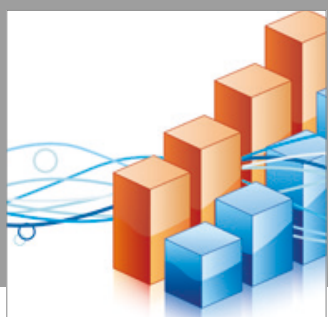

Advances in

Operations Research

vatersals

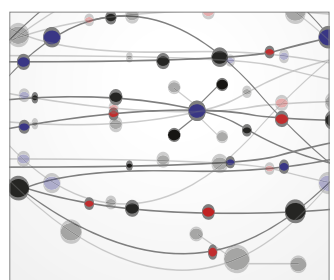

\section{The Scientific} World Journal
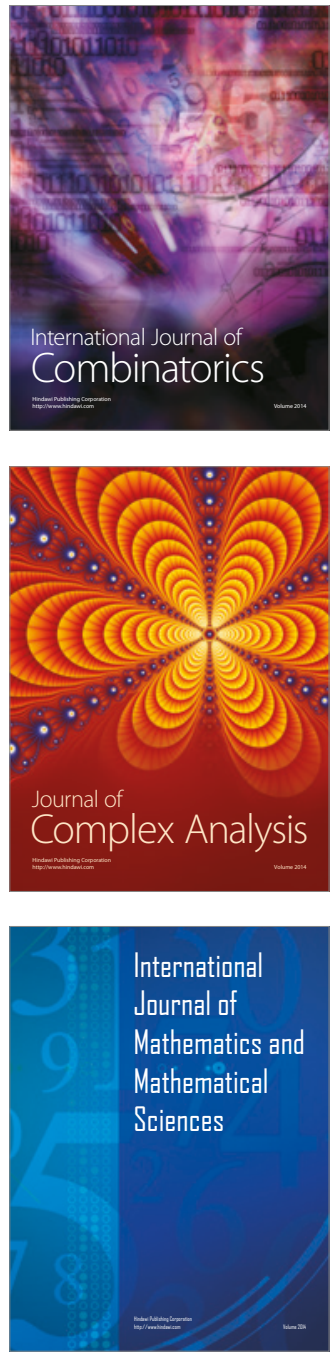
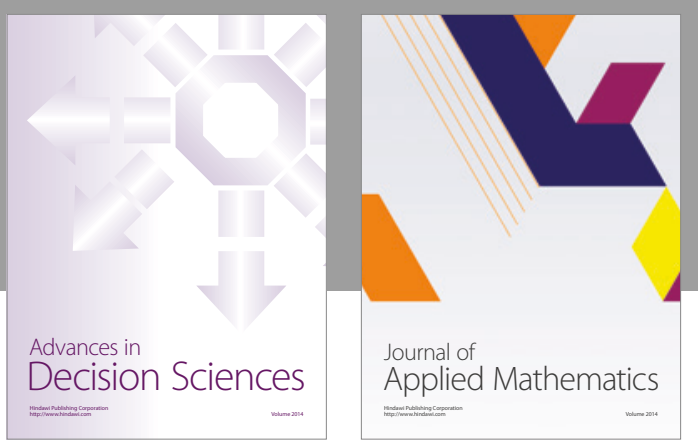

Algebra

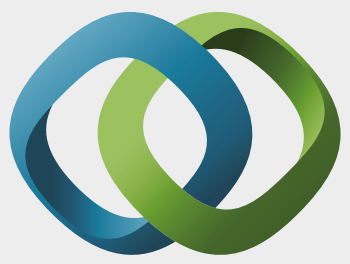

\section{Hindawi}

Submit your manuscripts at

https://www.hindawi.com
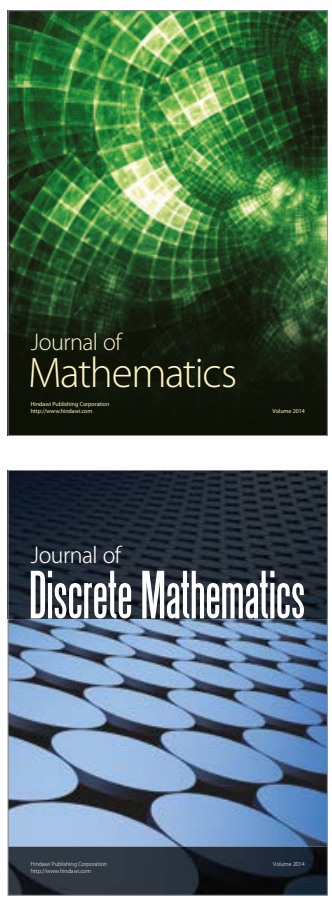

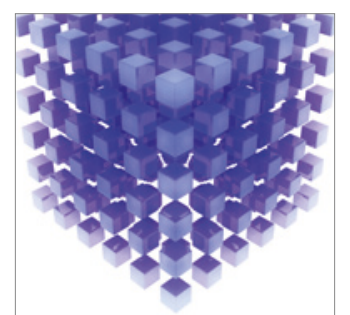

Mathematical Problems in Engineering
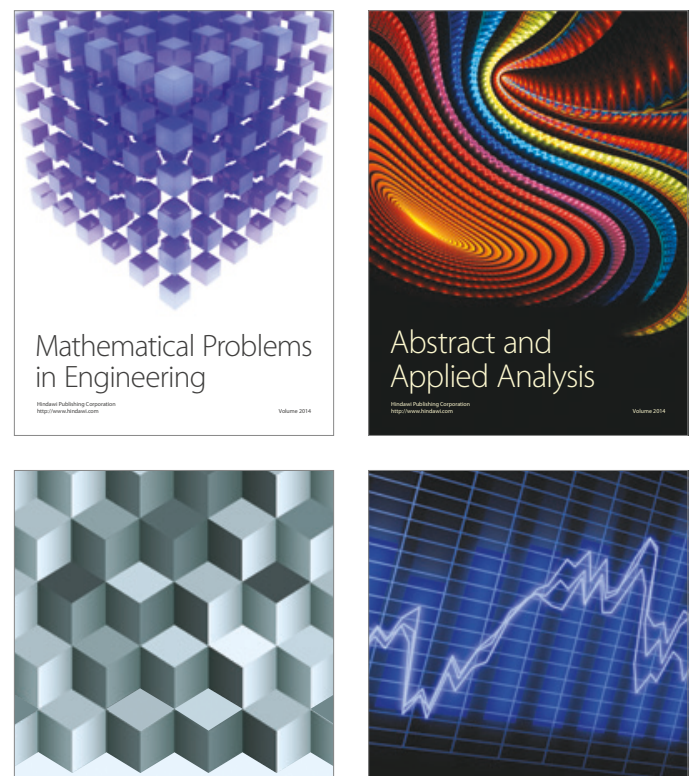

Journal of

Function Spaces

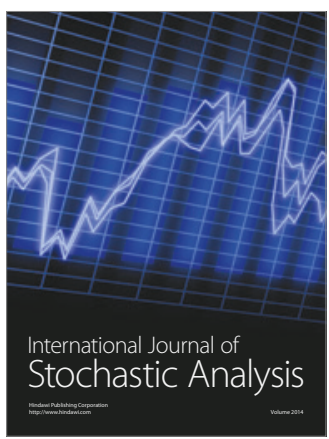

Probability and Statistics
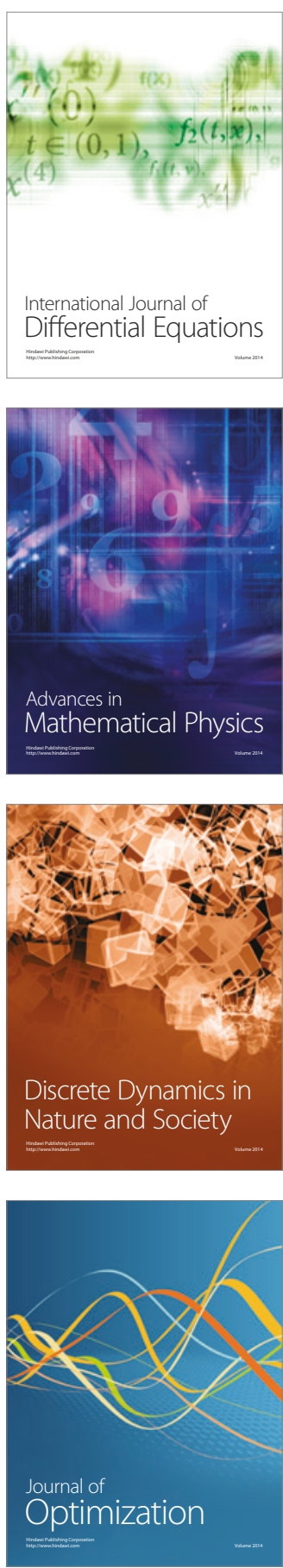